\title{
Schamanen gegen die Macht des Virus Verschwörungstheoriker*innen als Sozialfiguren der Corona-Pandemie
}

\author{
Erschienen in: Sozialfiguren der Corona-Pandemie \\ Von: Carolin Amlinger, Nicola Gess
}

Es ist Anfang Mai 2020, die WHO bittet auf einer Geberkonferenz um Gelder zur Entwicklung eines Corona-Impfstoffes. Derweil kursiert in Deutschland ein Video mit dem Titel "Gates kapert Deutschland". ${ }^{1}$ Es wird binnen weniger Tage über 5 Millionen Mal aufgerufen und findet regen Anklang bei den Demonstrant*innen, die sich in zahlreichen Großstädten gegen die Maßnahmen zur Einschränkung der CoronaPandemie versammeln. ${ }^{2}$ In dem Video sitzt ein glattrasierter Mann in hellblauem Hemd vor einem dicht bestückten Bücherregal, hinter ihm hängt gut sichtbar ein Plakat des Grundgesetzes, das die sogenannte "Querdenken"-Bewegung zu ihrem Symbol erhoben hat. Während sich der Rest des Raumes in Dunkelheit verliert, ist der Sprecher der folgenden Sätze hell ausgeleuchtet: "Mein Name ist Ken Jebsen. Ich bin ein freier Journalist in Deutschland und arbeite seit über 35 Jahren in diesem Land mit einem Presseausweis." Der dort spricht, weist sich selbst als Experte der öffentlichen Meinungsbildung aus. Er ist jener Journalist, der bis zum Jahr 2011 die Sendung KenFM beim rbb moderierte, bis der Radiosender sich wegen der Verletzung.journalistischer Standards von ihm trennte.

Jebsen umgibt sich seitdem mit der Aura eines Märtyrers. Er betreibt eine digitale Plattform, die einschneidende gesellschaftliche und politische Ereignisse, wie eben die Corona-Pandemie, mit geheimen Verschwörungen mächtiger Akteure in Verbindung bringt. ${ }^{3}$ In all dem verkörpert Jebsen das gängige Stereotyp eines Verschwörungstheoretikers: Gemeinhin stellt man sich darunter einen ressentimentgeladenen mittelalten Mann vor, der sich nach einem biographischen Bruch radikalisiert und einer alternativen Öffentlichkeit zugewendet hat, die seinen selbst ernannten Expertenstatus anerkennt. ${ }^{4}$ Der Anspruch, die "eigentliche" Wahrheit auszusprechen, wird von ihm, nicht selten auch aggressiv, gegen Kritiker*innen verteidigt.

Diese sozialfigurative Imagination von Verschwörungstheoretiker*innen ist eng verwandt mit denen der "Wutbürger" und zum Teil auch der "Maskenverweigerer"; sie ist aber keinesfalls mit innen identisch. Zwar wird das soziale Imago, das wir von Verschwörungstheoretiker*innen haben, nicht selten durch die Wirklichkeit konterkariert 
(erinnert sei an dieser Stelle an die 22-jährige "Jana aus Kassel", die sich während eines Corona-Protestes in Hannover mit Sophie Scholl verglich), dennoch artikuliert es über das verdichtete Bild bestimmte soziale Erfahrungen.

Die Corona-Pandemie ist ohne Zweifel eine krisenhafte soziale Erfahrung; eine existentielle Situation, die Angst macht und mit deren Erklärung und Bewältigung bis heute nicht nur Expert*innen und Politiker*innen ringen. Da wundert es wenig, dass Verschwörungstheorien, die Schuldige identifizieren und oft zugleich die Bedrohung durch das Virus herunterspielen, in der Corona-Krise eine neue Konjunktur erleben. ${ }^{5}$ Die "Mitte-Studie" von Andreas Zick und seinen Kolleg*innen, die antidemokratische Einstellungen in der deutschen Bevölkerung untersucht, kam bereits 2019 zu dem Ergebnis, dass Verschwörungstheorien einen subjektiv erfahrenen Kontrollverlust kompensieren. Sie reduzieren Komplexität, liefern anschauliche Erklärungen, bewältigen Kontingenz durch eine kohärente Sinndeutung und stiften Identität, die sich um die Freund-Feind-Figuration zentriert. Dabei ist der Glaube an Verschwörungstheorien häufig mit einem tiefen Misstrauen gegenüber "machtvollen Eliten" verbunden; auch die Figur des Drahtziehers ist allgegenwärtig. ${ }^{6}$ Entsprechend gibt auch Jebsen an, die wahren Köpfe hinter der Pandemie zu kennen: Die "Bill \& Melinda Gates Foundation [...] bestimmt das, was man aktuell Normalität nennt".

Verschwörungstheorien mögen sich als "Theorien" inszenieren, tatsächlich funktionieren sie jedoch wie Geschichten, mit den Verschwörungstheoretiker*innen als deren homodiegetische Erzähler*innen, d. h. sie sind selbst Bestandteil der erzählten Welt. Dabei greifen sie auf eines der ältesten und erfolgreichsten Basisnarrative überhaupt zurück - den Kampf zwischen Gut und Böse - und bauen es zu einem Plot aus, der am ehesten einem Spionageroman ähnelt und in dem sie selbst als mutige Aufklärer*innen und Widerstandskämpfer*innen eine Hauptrolle spielen. Jebsens Erzählung, die sich im Verlauf des Videos entspinnt, gleicht einer ungeheuerlichen Dystopie, die auch widersprüchliche Elemente miteinander verknüpft und sie in eine Handlungsfolge überführt, in der auch noch das kleinste Detail (wie z. B. ein Interview mit Gates in den Tagesthemen) eine Bedeutung für das Ganze hat: Das Ehepaar Gates steuere die weltweite Politik, wolle die Bevölkerung trotz unbekannter Risiken zwangsimpfen und chippen lassen, um so gemeinsam mit einer globalen Finanzelite die Weltbevölkerung zu kontrollieren. Entscheidend für die Überzeugungskraft von Verschwörungstheorien ist weder ihre faktische Beweisbarkeit noch ihre logische Widerspruchsfreiheit, sondern inre narrative Evidenz. Dabei sieht die typische Figurenkonstellation so aus: Auf der einen Seite die Verschwörungstheoretiker*innen, die im Namen des Guten gegen machtvolle Gegenspieler*innen, wie in diesem Fall Bill Gates (hier in dämonisierender Darstellung als Vampir), aber auch alle institutionalisierten Formen der Wissensproduktion und -kommunikation (wie z. B. die Virolog*innen des RKI) ankämpfen, die durch die dunklen Mächte infiltriert sind.

Um Glaubwürdigkeit zu produzieren, greifen Verschwörungstheoretiker*innen aber auch auf andere Verfahren zurück, die entdifferenzierend miteinander vermischt werden. So betreiben sie Mimikry an wissenschaftliche Theoriebildung, indem sie zum Beispiel eigene "Expert*innen" ins Feld führen und deren akademische Titel hervorheben, oder 
indem sie (extrem selektive) Belege für ihre Behauptungen sammeln oder, wie Jebsen, Floskeln wie "ich zitiere" oder "das kann man nachlesen" verwenden. Oftmals genügen schon allein diese Gesten, gepaart mit der In-Szene-Setzung von Empörung, um das Vertrauen eines Publikums zu gewinnen, das nach anschaulichen Erklärungen und einem Ausweg aus der gefühlten Ohnmacht sucht. Dann wieder wenden sie sich gegen die institutionelle Wissensordnung von Expert*innen und knüpfen an anekdotische Redeweisen an, die auf dem kollektiven Weitererzählen eines Augenzeugenberichts beruhen (“Ich möchte eine kurze Anekdote erzählen...", so Jebsen).

Das Nebeneinander ganz unterschiedlicher Formen der Produktion von Scheinevidenz erhöht die Anschlussfähigkeit von Verschwörungstheorien. Mithilfe von persönlichen Ansprachen stiften Verschwörungstheoretiker*innen eine Nähe zu Gleichgesinnten; durch die oben beschriebene Mimikry an "Theorie" drücken sie eine subjektiv wahrgenommene Überlegenheit aus. Sie versorgen ihre Anhänger*innen nämlich nicht nur mit einfachen Erklärungen, sie rüsten das fragile Subjekt auch mit Handlungsmacht aus und geben ihm ein Gefühl der Einzigartigkeit. Gerade angesichts ihrer öffentlichen Delegitimierung können sich Verschwörungstheoretiker*innen als heroische Subjekte fühlen. So ist die Verweigerung, eine Maske zu tragen oder Abstand zu halten, aus Sicht der verschwörungsaffinen "Querdenker*innen" eine Form des politischen Protests, der sich jedoch vor allem aus dem Unwillen speist, alte Überzeugungen (vom Glauben an eine geheime Weltverschwörung bis zur Skepsis gegenüber einem naturwissenschaftlichen Weltbild) fahren zu lassen. Dabei sind Verschwörungstheoretiker*innen keinesfalls per se ungebildet. ${ }^{7}$ Laut einer Studie aus dem Jahr 2020 von Oliver Nachtwey, Robert Schäfer und Nadine Frei sind die Teilnehmer*innen der Corona-Proteste mehrheitlich Angehörige der gebildeten Mittelschicht, die sich vom politischen System entfremdet haben und ein hohes Radikalisierungspotenzial aufweisen.

Dass ein signifikanter Zusammenhang zwischen dem Glauben an Verschwörungen und Gewaltbereitschaft besteht, konnte nicht nur die bereits erwähnte "Mitte-Studie" zeigen, sondern wurde spätestens am 6. Januar 2021 überdeutlich, als Trump-Anhänger*innen das Kapitol in Washington stürmten. Die Krawalle der Angreifer*innen, unter denen sich neben Rechtsextremist*innen und Antisemit*innen auch Mitglieder der chauvinistischen Proud Boys und der QAnon-Bewegung befanden, veranschaulichen die performative Kraft, die von der verschwörungstheoretischen Polarisierung von Freund und Feind ausgeht. Gleichzeitig wurde mit dem Sturm auf das Kapitol auch die Macht der Bilder im Verschwörungsglauben deutlich. Auf Social-Media-Plattformen zirkulieren nicht nur zahlreiche Verschwörungstheorien, sie gestalten diese unweigerlich mit. Die vielfach kostümierten Angreifer*innen, die das Geschehen mit ihren Smartphones dokumentierten, ähneln Spielfiguren aus einem Alternate Reality Game - nur dass das virtuelle Spiel nun in die Realität überführt wurde. Verschwörungstheorien, die sich erzähltheoretisch auch als faktuale Erzählungen mit fiktiven Inhalten beschreiben lassen, nähren sich aus eben dieser Diskrepanz (von faktual/fiktiv): das Fiktive soll Realität werden, die "alternate reality" ist bereits die einzig wahre. 
Die wohl eindrücklichste ikonische Figur der Kapitolstürmung war der sogenannte QAnon-Schamane, ein junger Mann mit blau-weiß-roter Gesichtsbemalung, bisonartigem Kopfschmuck und Kojotenfell, der eine Lanze mit US-Fahne in der Hand hielt - auf dem Screenshot zu sehen in einem Artikel der USA Today News, die bereits einen Tag später Hintergrundinformationen zu vielen "rioters" veröffentlichte .

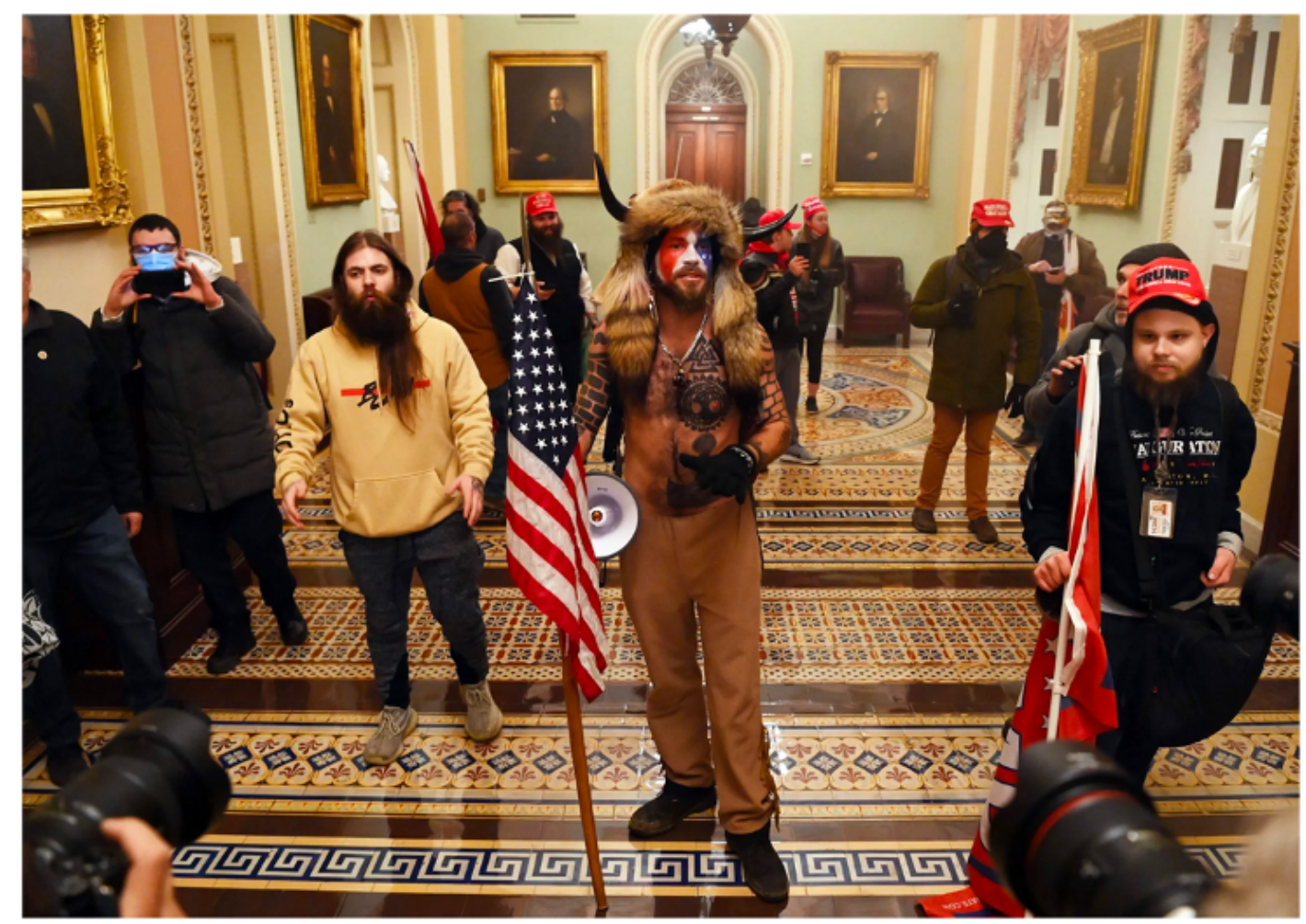

Jake Angeli, center in horned hat, is among supporters of President Donald Trump who breached security enter the Capitol on Jan. 6 as Congress meets ... Show more $\checkmark$ SAUL LOEB, AFP VIA GETTY IMAGES

Screenshot des Artikels "Capitol mob drew Trump supporters of all stripes, from a fireman to a 'QAnon shaman"' von Dinah Voyles Pulver \& Josh Salman in USA TODAY.

Quelle: https://eu.usatoday.com/story/news/2021/01/07/capitol-mob-drew-trump-fans-qanon-shamanfireman-jake-angeli-arrest/6585339002/

Andere Aufnahmen zeigen inn mit einem Plakat, auf dem "Q Sent Me" stand. Hinter dem Kostüm verbirgt sich der Youtuber Jake Angeli, ein esoterischer Influencer, dessen Pseudonym auf eine engelsgleiche Herkunft verweisen soll. In seinen selbstverlegten Büchern und Videos verbindet er schamanistische Thesen über Bewusstseinserweiterung mit einem ausgeprägten ökologischen Bewusstsein und Verschwörungstheorien. Auch die in der oben genannten Studie untersuchten Querdenker*innen wiesen eine starke Neigung zur Esoterik und dem Glauben an Selbstheilungskräften auf. Die Rede von Verschwörungsmythen statt -theorien ist in diesem Fall erhellend. Mythen sind in ihrem Ursprung sinnstiftende Erzählungen, die eine übermächtige und unverstandene Natur zu bewältigen versuchen. Ein Denken im Bann des Mythos geht davon aus, dass es unsichtbare Mächte gibt, die überall ihre 
Finger im Spiel haben; es löst die subjektiv erfahrene Ohnmacht über eine imaginative Figuration auf, in der unerklärliche Phänomene eine plastische Gestalt und dadurch eine benennbare Ursache erhalten. ${ }^{8}$

Der Gegenstand von Verschwörungstheorien ist jedoch nicht die Natur, sondern die moderne Gesellschaft, die offenbar als ebenso übermächtig und unverständlich, also als eine Art "zweite Natur", erlebt wird. Auf das Gefühl der Ohnmacht, aus der eine kritische Gesellschaftsanalyse erwachsen könnte, reagieren Verschwörungstheoretiker*innen mit einer quasi-mythischen Sinnkonstruktion, die einfache Erklärungen ("hinter allem steckt eine Gruppe böser Verschwörer*innen") und Handlungsoptionen liefert - auch im Fall der Corona-Krise, die als Ausdruck oder Projektionsfläche der gefühlten Ohnmacht dient, gegen die sich nun (in der Figuration von Bill Gates oder anderer vermeintlicher "Verschwörer*innen") gezielt ankämpfen lässt. Verschwörungstheorien verarbeiten das Gefühl, machtlos zu sein, und lösen diese Ohnmacht in eine Geschichte auf, in der nun die Erzähler*innen die Kontrolle über das Geschehen haben.

Die mit der Sozialfigur des Verschwörungstheoretikers verbundenen gesellschaftlichen Themen und sozialen Krisenerfahrungen können wie folgt zusammengefasst werden: Über sie wird der Vertrauensverlust in institutionalisierte Formen der Wissensproduktion und medialen Kommunikation artikuliert, aber auch ganz generell die Frage verhandelt, wie verbindliches und legitimes Wissen überhaupt zustande kommt. Zugleich wird über sie ein Ohnmachtsgefühl, eine Sehnsucht nach Handlungsmacht zum Ausdruck gebracht und damit indirekt auch die Frage nach politischer Partizipation und gesellschaftlichen Gestaltungsmöglichkeiten gestellt. Die Verschwörungstheorie selbst vermag all dies nicht einzulösen. Sie verliert sich stattdessen in den Raum des konspirativen Imaginären. Aber das Nachdenken über Verschwörungstheorien und die Sozialfigur des Verschwörungstheoretikers kann solche Perspektiven eröffnen.

\section{References}

1. Das Video kann hier eingesehen werden (letzter Aufruf: 26. Januar 2021).

2. Vgl. hier und im Folgenden zur detaillierten Analyse des Videos und der von Jebsen verbreiteten Verschwörungstheorien: Nicola Gess: Halbwahrheiten. Zur Manipulation von Wirklichkeit, Berlin 2021, S. 64-86.

3. Verschwörungstheorien artikulieren nach Karen M. Douglas und Kolleg*innen das Bemühen, "to explain the ultimate causes of significant social and political events and circumstances with claims of secret plots by two or more powerful actors" (4).

4. Vgl. zu diesem Stereotyp und seiner Infragestellung in der Forschung: Michael Butter: Nichts ist, wie es scheint. Über Verschwörungstheorien. Berlin 2018, S. 115-124.

5. Eine Studie von Jochen Roose kam 2020 zu dem Ergebnis, dass 30 Prozent der deutschen Bevölkerung Verschwörungstheorien für wahrscheinlich richtig und 11 Prozent für sicher richtig halten.

6. Die Nähe zum strukturellen Antisemitismus ist besonders offenkundig, die Michael Butter hier historisch rekonstruiert. 
7. Die "Mitte-Studie" konnte allerdings zeigen, dass mit steigendem Bildungsniveau die Zustimmung zu verschwörungstheoretischem Denken in der deutschen Bevölkerung abnimmt. Die Querdenker*innen der Corona-Proteste sind also sicherlich nochmals ein besonderer Fall.

8. Vgl. Norbert Elias: Engagement und Distanzierung, Frankfurt a. M. 1983.

SUGGESTED CITATION: Gess, Nicola; Amlinger, Carolin: Schamanen gegen die Macht des Virus. Verschwörungstheoriker*innen als Sozialfiguren der CoronaPandemie, in: KWI-BLOG, [https://blog.kulturwissenschaften.de/schamanen-gegen-diemacht-des-virus/], 15.02.2021

DOI: https://doi.org/10.37189/kwi-blog/20210215-0830 


\section{DuEPublico}

Duisburg-Essen Publications online

\section{$D_{E} U_{S} S_{S} S_{N}^{B}$ U R G}

offen im Denken

Dieser Text wird über DuEPublico, dem Dokumenten- und Publikationsserver der Universität Duisburg-Essen, zur Verfügung gestellt. Die hier veröffentlichte Version der EPublikation kann von einer eventuell ebenfalls veröffentlichten Verlagsversion abweichen.

DOI: $\quad$ 10.37189/kwi-blog/20210215-0830

URN: urn:nbn:de:hbz:464-20210215-102341-9

Alle Rechte vorbehalten. 